\title{
Análisis de la viabilidad empresarial en el preconcurso de acreedores
}

\author{
Analysis of corporate viability \\ in the pre-bankruptcy proceedings
}

\author{
Maria Jesus Segovia Vargas ${ }^{1, *}$ \\ Mara del Mar Camacho Miñano ${ }^{2}$ \\ ${ }^{1}$ Universidad Complutense de Madrid, España \\ ${ }^{2}$ Colegio Universitario de Estudios Financieros, España
}

Recibido el 22 de febrero de 2016; aceptado el 17 de octubre de 2016

Disponible en Internet el 11 de diciembre de 2017

\section{Resumen}

Las empresas en dificultades financieras pueden acogerse al preconcurso de acreedores, previo a su entrada en el proceso legal de insolvencias. El objetivo de este estudio es el posible diagnóstico de algunas características comunes que tienen las empresas con dificultades financieras para conseguir el éxito del preconcurso de acreedores, proponiendo el uso de metodologías de inteligencia artificial como complemento a los análisis tradicionales. Utilizando una muestra española de empresas en concurso y "sanas" se obtiene que los ratios de viabilidad financiera y del fondo de maniobra son determinantes para la efectividad del preconcurso. Nuestros resultados tienen importantes implicaciones para jueces, administradores concursales, auditores y gestores de empresas en concursos de acreedores.

Palabras clave: preconcurso acreedores, ley concursal, reorganización, insolvencia, metodologías de inteligencia artificial

Códigos JEL: G33, K22, M41, C44

\footnotetext{
*Autor para correspondencia.

Correo electrónico: mjsegovia@ccee.ucm.es (M. J. Segovia Vargas).

La revisión por pares es responsabilidad de la Universidad Nacional Autónoma de México.
} 


\begin{abstract}
Companies in financial difficulties can avail themselves of the pre-bankruptcy procedure, prior to its entry in the legal process of insolvency. The objective of this paper is to look for a possible diagnosis of some common distressed firm's characteristics in order to be successful at pre-bankruptcy procedure through artificial intelligence methodologies. Using a Spanish sample of bankrupt and healthy firms, our results show that financial viability and working capital ratios are fundamental for the effectiveness of pre-bankruptcy legal procedure. These findings may help to shed light on the implications for all the stakeholders involved in a pre-bankruptcy procedure.
\end{abstract}

Keywords: pre-bankruptcy legal procedure, insolvency act, reorganization, insolvency, artificial intelligence methodology.

JEL Classification: G33, K22, M41, C44

\title{
Introducción
}

Las situaciones de crisis económicas provocan cuantiosas pérdidas para la economía, en general y para las empresas, en particular. La insolvencia de empresas provoca una alteración significativa de cifras macroeconómicas tan relevantes para los países como el incremento de la tasa de desempleo, el aumento de la deuda pública en relación al producto interior bruto (PIB) o una disminución del crecimiento real del PIB. En estas circunstancias, el enfoque de las leyes concursales de cada país se vuelve esencial para el mercado, pudiendo incluso condicionan la economía nacional (LaPorta et al., 1998). De ahí que sea necesario hacer un esfuerzo en la posible predicción de sus causas y consecuencias (Du Jardin, 2015; Bauweraerts, 2016). Por ejemplo, la resolución de los concursos de acreedores es diferente dependiendo del país del que se trate, ya que existen diferentes leyes concursales en función de cada enfoque (Weijs, 2012; Georgescu y Baciu, 2014). Por lo tanto, el tipo de regulación concursal que se establezca en un país no es una decisión baladí. Hay estudios económicos que justifican cómo las leyes sobre la insolvencia de empresas pueden condicionar el desarrollo del emprendimiento en un país (Lee et al., 2011), el crecimiento económico nacional (Laporta et al., 1998), la estabilidad económica (Beraho, 2010) e incluso el desarrollo de los mercados de valores (Levine, 1998). De hecho, se demuestra empíricamente cómo una ley concursal u otra puede condicionar las inversiones extranjeras en un país (Pindado et al., 2008). Además, en países como España, en el que la dependencia bancaria para la financiación de las empresas es muy elevada (Krivogorsky et al, 2011), el problema de insolvencia se convierte en un problema global de la economía nacional. De ahí que sea de vital importancia un correcto desarrollo de la normativa sobre insolvencias para promover el crecimiento económico y la seguridad jurídica de los inversores.

El propósito de la Ley Concursal (LC) en España es tratar de resolver los problemas de viabilidad financiera de las empresas mediante la regulación de un procedimiento legal. Se trata de que empresas viables, pero con problemas de liquidez, se reorganicen y continúen en el mercado -mediante el procedimiento de convenio- y que las no viables, desaparezcan del mercado siendo liquidadas -mediante el procedimiento de liquidación- (Fernández, 2004). Sin embargo, la implantación de la LC, dadas las adversas situaciones del contexto económico mundial actual, no ha sido todo lo eficiente que el legislador hubiese deseado. Según el anuario 
de estadística concursal del año $2011^{3}$, alrededor de 93 empresas de cada 100 que entran a concurso se liquidan.

En la LC española, al igual que en otras legislaciones a nivel internacional, el procedimiento legal se divide en tres fases: el 'preconcurso' o propuesta anticipada de convenio, la fase común y la fase sucesiva. Para favorecer las refinanciaciones, reducir los costes del procedimiento concursal, y facilitar los trámites del proceso se modificó la LC en el año 2009, en el año 2011 y recientemente con el RD 4/2014, con la finalidad de hacer más efectivo el 'preconcurso', objeto de análisis de este artículo. Antes de solicitar el inicio del proceso judicial, se intentan buscar soluciones, tales como una refinanciación privada, para poder seguir sobreviviendo en el mercado sin el lastre de la etiqueta "en concurso". Para evitar esos problemas, se intenta reducir el proceso y mejorar su eficiencia con nuevas herramientas, tales como acuerdos de refinanciación y escudos protectores (Pulgar Ezquerra, 2011).

El 'preconcurso' se basa en una comunicación "extrajudicial” (con abogado y procurador) de la empresa para informar al Juzgado de que se han iniciado negociaciones para alcanzar un acuerdo de refinanciación o adelantar una propuesta anticipada de convenio. Este proceso ha estado sujeto a diversas modificaciones legales para adaptarse a la realidad económica del momento (Olivencia Ruiz, 2015). Su finalidad última es "prevenir" que la crisis empresarial no sea irreversible, con medidas que ayuden a conocer anticipadamente la situación del negocio y poder tomar medidas idóneas de saneamiento (Fernández del Pozo, 2010). De este modo, se concede una prórroga a empresas que se encuentran en situación de insolvencia a corto plazo ya que la ley suspende la obligación del deudor de presentar el concurso voluntario durante un plazo máximo de cuatro meses. Este procedimiento también bloquea la presentación del concurso obligatorio por parte de sus acreedores. Para que una empresa en dificultades pueda acceder a la fase preconcursal debe cumplir dos requisitos: haber iniciado negociaciones para obtener un posible convenio anticipado y, en un plazo de dos meses, informar al juzgado competente.

Durante el período preconcursal, los deudores obtienen indudables beneficios ya que la empresa puede buscar refinanciar su deuda y evitar así el proceso concursal o puede planificar un posible convenio cuando entre en el proceso concursal o bien instar un concurso voluntario sin las tensiones propias de situaciones "in extremis". Sin embargo, la fase preconcursal también puede provocar perjuicios a los acreedores si no se realiza de manera correcta ya que pueden ver reducidas sus expectativas de cobro o demorarse mucho en el tiempo (Hernández Arranz, 2009). En la práctica, la implementación del proceso preconcursal se ha visto cuestionada porque algunas empresas (caso Rumasa ${ }^{4}$ y sus filiales, por ejemplo) han utilizado este procedimiento extrajudicial para que sus gestores evitaran el riesgo de concurso obligatorio y mantenerse en la gestión, y, en ocasiones, poder descapitalizarla.

Teniendo en cuenta lo anteriormente expuesto, el objetivo de este estudio es analizar desde un punto de vista empírico qué características comunes tienen las empresas asociadas al éxito de su reorganización en una situación de crisis, con la finalidad de "diagnosticar" en qué compañías sería efectivo el preconcurso y en cuáles el preconcurso es una "situación de coma inducido", con el consiguiente perjuicio para sus clientes, acreedores e incluso accionistas, y

\footnotetext{
${ }^{3}$ Puede consultarse en https://www.registradores.org/estaticasm/Estadistica/concursal/Estadistica_Concursal_Anuario.pdf

${ }^{4}$ Para conocer este caso véase, entre otras, las siguientes noticias, entre otras: http://www.europapress.es/economia/ noticia-nueva-rumasa-pide-preconcurso-diez-empresas-20110217191039.html ; http://www.cincodias.com/articulo/ empresas/nueva-rumasa-sumaria-29-empresas-preconcurso-incluir-hoteles/20110303cdscdiemp_15/.
} 
debería iniciarse el concurso por "vía de urgencia". Para ello se utilizan los estados financieros de todas las empresas en concurso de acreedores acaecidos durante el año 2010 en la Comunidad de Madrid, así como, una muestra de empresas sanas. Se ha seleccionado el año 2010 porque es el momento en el que entró en vigor la reforma introducida por el Real Decreto Ley 3/2009 de 27 de Marzo que permitió la declaración del preconcurso de acreedores. En definitiva, dado que este articulo viene a analizar el impacto de esta figura jurídica, se escogió el año en que se produce el cambio de legislación.

La intensidad de la crisis financiera ha dado lugar a la aparición de literatura que trata de dar respuesta al objetivo planteado con métodos paramétricos de tipo estadístico. Sin embargo en los últimos años para la resolución de problemas tanto a nivel micro como a nivel macro se ha acudido a herramientas procedentes de tanto de la biología, como la física o como en nuestro caso, a la inteligencia artificial. Por tanto para lograr el objetivo expuesto se utilizarán metodologías basadas en la inteligencia artificial (árboles de decisión) modelizando las características de las empresas siguiendo un criterio de solvencia, lo que supondrá un método, entre otros, para garantizar la continuidad de una empresa en el mercado.

Las principales contribuciones de este estudio son tres. Primera, se trata de un estudio empírico sobre aspectos económico-financieros de empresas concursadas. Son escasas las investigaciones empíricas realizadas en este campo (Saez-Santurtun, 2010), sobre todo, por la dificultad de acceso a la información necesaria en muchas legislaciones. Segunda, se exponen algunas características económico-financieras que condicionan la viabilidad del preconcurso, lo que ayudaría a terceros interesados a adoptar decisiones de una manera objetiva. Tercera, se trata de promover la aplicación de métodos alternativos (árboles de decisión) distintos a los tradicionalmente utilizados (técnicas estadísticas) en las ciencias sociales en general y a este problema, en particular que permite superar algunos de los inconvenientes presentes en las metodologías más tradicionales, o quizás ofrecer otra visión del mismo problema.

El artículo se estructura de la siguiente manera: en primer lugar, se expone el procedimiento legal de concurso de acreedores en España, analizando sus diferencias y similitudes con México. A continuación, se plantea la investigación, con el desarrollo de la muestra, las variables del estudio y la metodología empleada. Tras los resultados y su discusión, se plantean las principales conclusiones del presente trabajo de investigación.

\section{El sistema concursal español}

La LC en España está regulada por la ley 22/2003, que entró en vigor en septiembre del año 2004 y que ha sido modificada en sucesivas ocasiones recientemente como consecuencia del impacto de la crisis económica mundial y europea. Además, dicha ley introdujo la creación de unos juzgados mercantiles especializados. En línea con leyes europeas similares, la principal característica de la LC es que unifica en un solo procedimiento legal todo el proceso de insolvencia judicial, denominado "concurso de acreedores". Un esquema de la ley concursal puede verse en la Figura 1. 


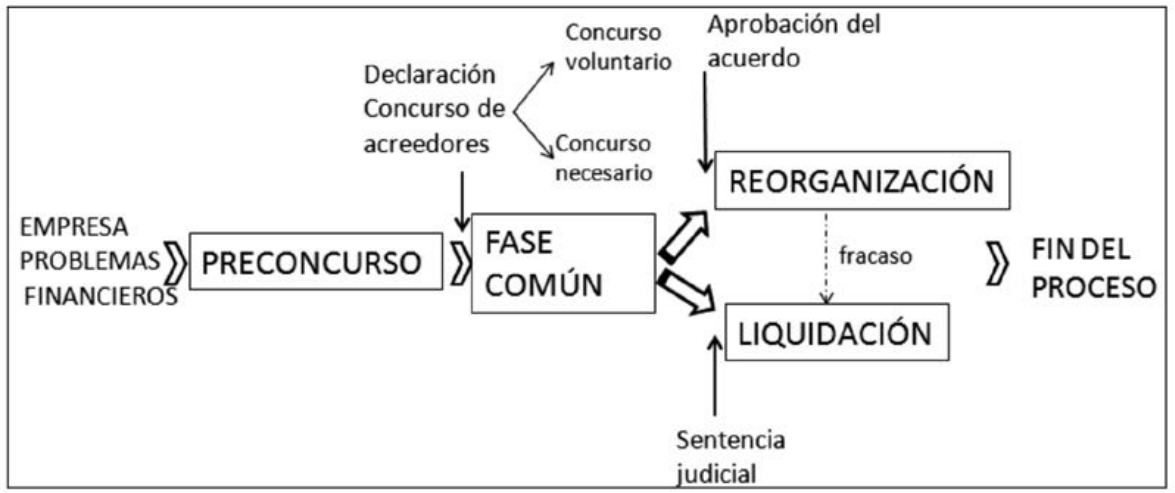

Figura 1. Esquema del proceso concursal español. Fuente: Elaboración Propia

La LC española permite, inspirada en el derecho mercantil italiano, un mecanismo de prevención del concurso mediante la negociación entre las partes interesadas, antes de la declaración de concurso para intentar la supervivencia de la empresa en el mercado. En muchas ocasiones, el concurso supone un lastre en sí mismo para que las empresas sobrevivan. Por ejemplo, se ve mermada la confianza con terceros cuando dichas empresas tienen que presentar sus estados financieros señalando expresamente la etiqueta de "en concurso de acreedores". Una empresa puede solicitar el concurso siempre que se pruebe que es solvente y que, sólo temporalmente, es incapaz de hacer frente a sus obligaciones financieras. Consecuentemente, un acuerdo de reorganización o convenio anticipado supone para los gestores que se reduce en el tiempo la probabilidad de liquidación, la mala imagen, costes en ocasiones innecesarios y burocracia que sucede tras una reorganización de convenio tradicional.

Si una empresa no puede acogerse a la fase de preconcurso, se inicia el proceso concursal con la declaración de insolvencia ante el juez y comienza la llamada "fase común", donde los gerentes y los administradores concursales (jueces, economistas, auditores, acreedores...) se dan cita ante el juez para analizar la situación económico-financiera de la empresa fracasada. Dicho procedimiento puede ser obligatorio cuando son los acreedores los que acuden al juez o voluntario cuando son los propios directivos o gestores de la empresa la que acude al juzgado y declara que no puede hacer frente a sus obligaciones de pago (artículo 2 de la LC). La distinción entre concurso voluntario y obligatorio es importante porque en el primer caso los gestores pueden seguir dirigiendo la empresa, que en el caso de un concurso obligatorio serán substituidos por los administradores concursales que señale el juez a cargo del concurso. La fase común finaliza cuando, una vez remitido el informe de los administradores concursales al juzgado, se establece el acuerdo de convenio o liquidación entre todas las partes interesadas en el concurso. Se empieza la última fase del concurso o fase sucesiva. Una vez que el juzgado ha aceptado el convenio, los acreedores y la empresa pueden negociar los términos de la reorganización empresarial. Si no existe acuerdo o la empresa no es solvente o no tiene futuro para continuar desarrollando su actividad, se inicia su liquidación. Por lo tanto, el propósito del convenio es preservar el negocio y permitir a la empresa continuar en el mercado. El propósito de la liquidación es que se vendan todos los activos de la empresa y con el dinero conseguido 
se puedan satisfacer los derechos de cobro de los acreedores, finalizando la vida de la empresa. Una vez que se cumplen los requerimientos del juzgado, el procedimiento legal del concurso finaliza.

En cualquier caso, es evidente que existen diferencias significativas en el impacto que ambos resultados tienen para la empresa y sus gestores, empleados, proveedores, clientes y todos los terceros interesados en la misma. Un procedimiento legal eficiente debería analizar correctamente el resultado final más adecuado. Es decir, una decisión de liquidación debería producirse cuando existen problemas económico-financieros difíciles de resolver, con prácticas de manipulación de los resultados, viéndose comprometida la integridad de la gestión y cuando la oportunidad de conseguir éxito sea remota. Por otra parte, aquellas empresas que hayan experimentado problemas financieros temporales, debido a una recesión económica, a la falta de dinamismo del sector y/o la implementación de malas estrategias de gestión pero que, una vez reorganizada, la empresa pueda volver a desarrollar su actividad sin problemas, habiendo reenfocado su misión, estrategia, gestión y estructura financiera totalmente.

\section{El sistema concursal mexicano}

La crisis económica actual ha supuesto un cambio de contexto fundamental para la supervivencia o no de las empresas en México. De hecho, algunas empresas mexicanas han infravalorado, al igual que en anteriores crisis (Watkins, 2003), el impacto de ese contexto económico adverso en sus estados financieros y la gestión de riesgos asumidos por dichas empresas se ha vuelto clave para su futuro. Una mala gestión de los riesgos del contexto económico ha supuesto que muchas hayan entrado en el procedimiento legal de insolvencia, denominado "Concurso mercantil", ya sea a petición propia o bien por la demanda de alguno de sus acreedores. Y al igual que en el mencionado caso español, el número de concursos declarados en México ha crecido de manera significativa, según el Informe Labores IFECOM.

La actual Ley de Concursos Mercantiles en México (LCM en adelante) fue promulgada y publicada el 12 de Mayo del año 2000 en el Diario Oficial de la Federación (DOF), derogando la hasta entonces Ley de Quiebras y suspensión de pagos de 1943. El origen de la LCM tuvo dos finalidades, por un lado, reducir los abusos procesales a los que se prestaba el procedimiento de la Ley de Quiebras y Suspensión de Pagos, y, por otro lado, la introducción de una nueva corriente en el tratamiento a los problemas de insolvencia en materia mercantil (Rojas Vertiz, 2002). Entre los objetivos de la nueva ley estaría preservar el valor de las empresas en situación de estrés financiero facilitando un arreglo voluntario entre los deudores y los acreedores y propiciando soluciones extrajudiciales. Lo que se pretende es conseguir un plan viable de reorganización o, de otra manera, proveer un ordenado y eficiente proceso de liquidación, maximizando el valor de los activos y distribuirlo legalmente entre los distintos acreedores.

Las etapas del Concurso en México son tres: la etapa anterior al concurso, la conciliación y la quiebra. La primera etapa es imprescindible, la segunda y la tercera pueden ser alternativas. La etapa de conciliación tiene como finalidad procurar que el comerciante y sus acreedores reconocidos lleguen a un acuerdo respecto de los términos y condiciones conforme a los cuales el comerciante pagará sus obligaciones de pago. Comienza cuando el comerciante es declarado en concurso mercantil. La etapa de quiebra, cuyo objetivo es la liquidación consistirá en vender la totalidad de sus bienes con la finalidad de pagar sus deudas. De manera previa a la etapa 
de conciliación o quiebra, debe determinarse la existencia del propio concurso mercantil. Es decir, existiría una "etapa previa" en la cual se determinaría la insolvencia. Para ello, el juez ordena a un verificador que, mediante un análisis o auditoría, determine si de la condición financiera de la empresa se desprende insolvencia de conformidad con los criterios que la propia LCM establece. Es decir, el objetivo fundamental de la llamada visita de verificación consiste en comprobar que se cumplen los requisitos establecidos por la ley para la fase pre concursal. Esta situación permite a las compañías con problemas financieros presentar un plan de reestructuración previa, haciendo que la reorganización del negocio quede abierta en la etapa de la conciliación. Por lo tanto, entendemos que se trata de un procedimiento preconcursal similar al caso español ya que su principal objetivo coincide, es decir, poder comenzar la reestructuración de la empresa en la etapa de la conciliación (Contreras et al., 2015). Esta importante mejora realizada a la actual LCM se produjo en el año 2007 al introducirse el título décimo cuarto (artículos 339-342).

Por tanto, en el caso mexicano, al igual que en el caso español, sería deseable contar con metodologías adicionales y objetivas que permitan ver qué patrones o características tienen las empresas que superarían con éxito el concurso de acreedores y que podrían ayudaran al verificador previsto en la LCM a la toma de decisiones eficaces. En otras palabras, se trataría de que, de una manera rápida y poco costosa, se conocieran qué empresas son viables económicamente y se puedan reorganizar financieramente y qué empresas son no viables económicamente, proponiendo su liquidación lo más rápido posible.

\section{Estudio empírico para el diagnóstico del exito del preconcurso.}

La viabilidad del proceso preconcursal va a tener importantes implicaciones para todas las partes interesadas en el mismo, entre las que destacan la posibilidad del ahorro de costes y de tiempo. Disponer de una herramienta sencilla, objetiva, rápida y precisa para la discriminación de en qué empresas el preconcurso permitiría su supervivencia en el mercado es vital para gestores de empresas, administradores concursales, acreedores y jueces. Con el estudio empírico que proponemos tratamos de responder a la pregunta siguiente: ¿Es viable que una empresa concreta, con determinadas características, se acoja a un proceso preconcursal?

Existen diversos estudios que analizan la predicción de insolvencia de las empresas (véase una revisión de la literatura en Tascón y Castaño, 2012 o Altman et al., 2016) y también hay literatura sobre las diferentes metodologías tanto tradicionales (véase Bauweraerts, 2016) como de inteligencia artificial aplicadas a este problema (entre otros, Kumar y Ravi, 2007; Chaudhuri, 2013). Sin embargo, no se han encontrado literatura relativa a la predicción de situaciones de insolvencia previas al concurso de manera específica, objeto de este estudio.

\section{Muestra del estudio}

El estudio empírico propuesto está formado por 1,713 empresas, de las cuales 799 son empresas sanas y 914 son empresas que han entrado en el proceso concursal. El proceso de elaboración de la muestra se inició con la selección de todas las empresas en concurso de acreedores en los doce juzgados de lo Mercantil de la Comunidad de Madrid, extraídas de 
la página web de la Asociación de Registradores de España (www.publicidadconcursal.es) desde mayo a julio del año 2010. En paralelo, se seleccionaron una muestra de empresas sanas teniendo en cuenta el sector, la forma jurídica y los años que presentan sus estados financieros. Una vez seleccionadas todas empresas, se ha ido buscando, según su CIF, la información económico-financiera disponible en la base de datos $\mathrm{SABI}^{5}$. Se han eliminado aquellas para las que no había información disponible.

\section{Variables del estudio}

Según la información de la base de datos y de otros estudios previos sobre predicción de insolvencias (Bhirmani et al. 2010, Tascón y Castaño, 2012, entre otros), en la Tabla 1 se exponen las variables o características de las empresas utilizadas para este estudio.

Tabla 1.- Variables seleccionadas para nuestro estudio

\begin{tabular}{|c|c|c|}
\hline VARIABLE & Explicación & Código WEKA \\
\hline Forma jurídica & Sociedad anónima y sociedad limitada & Legal_Form \\
\hline Número de accionistas & & NSh \\
\hline Número de filiales & & NSt \\
\hline Sector & Construcción, industria, comercio y servicios & sector \\
\hline $\begin{array}{l}\% \text { activos no corrientes } \\
\text { sobre total activos }\end{array}$ & $=$ Activos no corrientes $/$ total activos & NCA_to_TA \\
\hline $\begin{array}{l}\text { Rentabilidad } \\
\text { de los activos (ROA) }\end{array}$ & $=$ Beneficio operativo/total activos & ROA \\
\hline $\begin{array}{l}\text { Ratio de viabilidad } \\
\text { financiera }\end{array}$ & $=$ Gastos por intereses/EBITDA & Financial Viability ratio \\
\hline Tamaño de la empresa & $=$ Logaritmo del total activos & Size \\
\hline Ventas & Importe neto de la cifra de negocios & Sales \\
\hline EBITDA & $\begin{array}{l}=\text { Resultado antes de intereses } \\
\text { y amortización de activos }\end{array}$ & EBITDA \\
\hline Ratio fondo de maniobra & $=($ Activo corriente- pasivo corriente $) /$ total activos & WC_to_TA \\
\hline $\begin{array}{l}\% \text { Patrimonio neto } \\
\text { sobre total pasivos }\end{array}$ & $=$ Patrimonio neto/total pasivos & ETL \\
\hline Rotación de activos & $=$ Ventas/total activos & ASSET_TURNOVER \\
\hline $\begin{array}{l}\text { Ratio de independencia } \\
\text { financiera }\end{array}$ & $=$ Patrimonio neto/total activos & FINANCIAL_INDEP \\
\hline $\begin{array}{l}\text { Ratio de cobertura } \\
\text { de intereses* }\end{array}$ & $\begin{array}{l}=\text { Beneficio antes de intereses e impuestos/ } \\
\text { intereses }\end{array}$ & DISTRESS_CRITERIO \\
\hline Ratio de test ácido* & $=($ Activo corriente - inventario $) /$ pasivos corrientes & cash \\
\hline Ratio de garantía & $=$ Activo total $/$ pasivo total & guaranty \\
\hline \multicolumn{3}{|l|}{ VARIABLE DE DECISIÓN } \\
\hline Ratio de solvencia* & =Activo corriente/pasivo corriente & insolvency \\
\hline
\end{tabular}

Fuente: Elaboración Propia

*Variables categorizadas con valores 1 ó 2 .

\footnotetext{
${ }^{5}$ Puede verse más información en http://www.informa.es/informa/index.php/en/Financial-products/SABI-AMADEUS-y-ORBIS
} 
Destacar que hay algunas variables que se han categorizado, es decir, se les ha asignado valor 1 o valor 2 en función de que cumplan algunas características. Estas características son los valores que generalmente son tomados como referencia en los análisis contables (véase Rivero Romero, 2009). Estas variables están señaladas con un asterisco (*) en la Tabla 1 y son:

-ratio de cobertura de intereses: tomando valor 1 si el ratio de cobertura de intereses es inferior a cero y tomando valor 2 cuando el ratio de cobertura de intereses sea igual o superior a cero. Es decir, si la empresa puede pagar sus intereses con los beneficios generados antes de intereses e impuestos, la variable tomará valor 2 , y 1 en caso contrario.

-ratio de test ácido: tomando valor 1 si el ratio es inferior a uno y tomando valor 2 cuando dicho ratio sea igual o superior a uno. Empresas con ratios de test ácido inferior a uno no pueden pagar sus pasivos corrientes con la liquidez y los derechos de cobro que posee, y su viabilidad debe ser analizada con precaución.

-ratio de garantía: tomando valor 1 si el ratio es inferior a 2 y tomando valor 2 cuando dicho ratio es igual o inferior a 2 . Se considera este valor como ideal para la continuidad de la empresa en el mercado.

-ratio de solvencia: tomando valor 1 si el ratio es inferior a 1.5, considerándose la empresa no solvente. El ratio toma valor 2 si su cuantía es igual o superior a 1.5. Se considera que el ratio de solvencia es ideal cuando la cuantía del activo corriente es un 50\% mayor que la del pasivo corriente, según la literatura tradicional de ratios. Este ratio es considerado la variable de decisión de nuestro estudio ya que hay diferentes estudios que justifican su uso en la predicción de insolvencia (entre otros, véase: Bryan et al., 2002; Mongrut et al., 2011; Rose-Green and Lovata, 2013)

\section{Metodología}

Asistimos en la actualidad a un nuevo enfoque a la hora de abordar muchos problemas financieros basado en la aplicación de técnicas y metodologías no paramétricas encuadradas en el ámbito de la Inteligencia Artificial (IA). Las técnicas paramétricas, fundamentalmente estadísticas que tradicionalmente se utilizan, presentan resultados satisfactorios pero tienen la limitación de que muchas de las hipótesis estadísticas requeridas al modelo o a la distribución que siguen datos para estimar los parámetros (por ejemplo, si existen o no observaciones atípicas) no se cumplen cuando se emplean datos reales. Además, los resultados son complejos de interpretar para un usuario no experto en dichas técnicas. Las técnicas de IA, al no requerir que los datos satisfagan ningún tipo de hipótesis pueden superar esas limitaciones y en cualquier caso, pueden ser usadas de manera complementaria para dar un nuevo enfoque al problema que se quiere abordar. En problemas de insolvencia y predicción de riesgos estas metodologías han sido testadas con anterioridad (Kumar and Ravi, 2007; Wu; 2010; Chen, 2011; Koyuncugil y Ozgulbas, 2012, Du Jardin, 2015 entre otros).

Dentro de la IA, el aprendizaje automático (machine learning) consiste en la elaboración de programas de ordenador que sean capaces de generar conocimiento a través del análisis de los datos y posteriormente utilizar dicho conocimiento para realizar deducciones sobre nuevos datos. Dentro de las técnicas de este enfoque encontramos aplicados a datos españoles: redes neuronales artificiales o más recientemente maquinas de vectores soporte (Gutierrez et 
al., 2010), algoritmos de inducción de reglas (Sanchis et al., 2007), metodología "rough set" (Camacho-Miñano et al., 2015) y árboles de decisión (Diaz et al., 2009). Algunas de ellas tienen un carácter explicativo (inducción de reglas y árboles de decisión), otras se caracterizan por un enfoque de caja negra (black box), como las redes neuronales, en las que es difícil explicar el conocimiento que se extrae de su utilización. Dada la naturaleza del problema que queremos tratar, utilizaremos técnicas explicativas. En concreto se utilizará el árbol de decisión C4.5 dado que posiblemente sea de los árboles de decisión más flexibles y utilizados en muy diferentes ámbitos y también en relación con las quiebra o insolvencia de las empresas (entre otros recientemente, Abellán y Mantas, 2014; Serrano-Cinca y Gutiérrez-Nieto, 2013; Sathyadevan y Nair, 2015).

El algoritmo J48 es la implementación en Java de libre acceso del algoritmo C4.5 que contiene WEKA. WEKA es el paquete de minería de datos desarrollado por la Universidad de Waikato (Witten y Frank, 2005; Bouckaert et al., 2015) con el que hemos desarrollado el análisis del algoritmo mencionado. A continuación y de manera resumida, indicaremos las características esenciales de esta metodología.

La metodología C4.5, como cualquier árbol de decisión, es un modo de representar los patrones que subyacen en un conjunto de datos, en forma de un conjunto de condiciones exhaustivas y mutuamente excluyentes entre sí. Esas condiciones se organizan conforme a una estructura arborescente compuesta por nodos que se conectan a través de ramas. Los arboles de decisión se utilizan de la siguiente forma: para obtener una predicción o clasificación cada caso o ejemplo es filtrado bajando por el árbol, comenzando desde el nodo raíz, hasta alcanzar una hoja (en este trabajo un ejemplo será una empresa estudiada para las variables señaladas en la Tabla 1). En cada nodo, el ejemplo se testa y, en función del resultado de dicho test, continúa su recorrido por el árbol. La predicción es la clase que señala la hoja en la que ha terminado dicho caso o ejemplo una vez que ha recorrido el árbol.

El modo en que se generan los arboles se basa en distintos algoritmos de aprendizaje. Pero todos ellos generan la estructura de árbol mediante la realización de particiones recursivas de arriba a abajo cada vez más pequeñas del conjunto de los datos. Es decir, los datos comenzando por el nodo raíz, en cada nodo, en función del resultado del test se dividen y así sucesivamente obteniendo subconjuntos de los mismos. El proceso finaliza cuando los subconjuntos son puros o cuando su pureza no puede incrementarse. Un subconjunto es puro cuando solo contiene ejemplos que pertenecen a una clase. El objetivo es realizar el menor número de particiones posible para que el árbol resultante sea pequeño y el conjunto de ejemplos que verifiquen cada clase sea muy grande.

Para la generación de árboles se han desarrollado diversos algoritmos de aprendizaje y en cada nodo dicho algoritmo determina la partición más adecuada. Entre ellos, el algoritmo más conocido y utilizado se encuentra el desarrollado por Quinlan (1993) e implantado en el C4.5 (Quinlan, 2014).

En el C4.5, el criterio utilizado para hacer las particiones se apoya en una serie de conceptos procedentes de la Teoría de la Información. Parte, al igual que otros algoritmos, de la premisa de tomar en cada rama del árbol para hacer la correspondiente partición aquella variable que proporciona más información de cara a clasificar los elementos que constituyen el conjunto de entrenamiento o conjunto de datos usados para construir el árbol. Para establecer la variable que proporciona la mayor información, en el caso del C4.5 se emplea el ratio de ganancia (Gain 
Ratio $\frac{I x ; y_{i}}{H y_{i}}$, , esto es, el porcentaje de la información proporcionada por $y_{i}$ que es útil para conocer $\mathrm{x} .^{6}$

\section{Resultados empíricos obtenidos}

El árbol C4.5 está indicado para analizar problemas de clasificación de objetos con múltiples variables, que consisten en asignar una serie de objetos señalados por dichas variables a una categoría previamente definida. Se parte de un conjunto de empresas caracterizadas por los valores de una serie de variables económico-financieras y legales y tratamos de asignarlas a una de estas dos categorías: solventes o insolventes. Cabe recordar que el criterio utilizado para clasificar las empresas en solventes o insolventes es el ratio de solvencia a corto plazo, tal y como se ha justificado con anterioridad. El ratio de solvencia a corto es el cociente entre el total de activos a corto plazo y el total de deudas a corto plazo, es decir, nos determina la capacidad de una empresa de poder pagar sus deudas con vencimiento inferior a doce meses con los recursos disponibles en el mismo período.

Para la aplicación de la metodología, primeramente, hemos construido dos tablas de información, una para las empresas sanas y otra para las concursadas, para las cuales hubiese información completa para todas las variables consideradas. La primera de ellas contiene 799 empresas sanas, de las cuales han sido 422 son solventes (clase 2) y 377 son insolventes (clase 1). La segunda tabla está formada por 914 empresas concursadas, de las cuales 113 son solventes (clase 2) y 801 son insolventes (clase 1). Esto significa que hay 377 empresas sanas con problemas financieros y 113 empresas en concurso sin graves problemas financieros, que ambas podrían iniciar el proceso de preconcurso. Las primeras son insolventes y, aunque siguen en el mercado, podrían reorganizarse para evitar una posible entrada en el proceso concursal que "lastrara" su futuro. Las empresas en concurso que son solventes podrían reorganizarse en preconcurso, sin iniciar el proceso concursal porque pueden pagar sus deudas y evitar, de este modo, el impacto negativo del proceso concursal: desconfianza de los clientes y proveedores, mala imagen comercial, posibles problemas con el suministro de mercaderías, etc.

Ambas empresas, sanas y concursadas, han sido evaluadas de acuerdo con los valores que toman las 18 variables económico-financieras y legales seleccionadas y expuestas en la Tabla 1.

Las dos tablas se han introducido en WEKA con el que hemos desarrollado el análisis del C4.5.

En primer lugar, vamos analizar el modelo obtenido por la metodología C4.5 para las empresas sanas, según se observa en la Figura 2. Dicho árbol tiene un porcentaje de aciertos del $97 \%$ lo cual justifica su interpretación.

\footnotetext{
${ }^{6}$ Para ver más información sobre estos conceptos consúltese Quinlan (1993, 2014).
} 


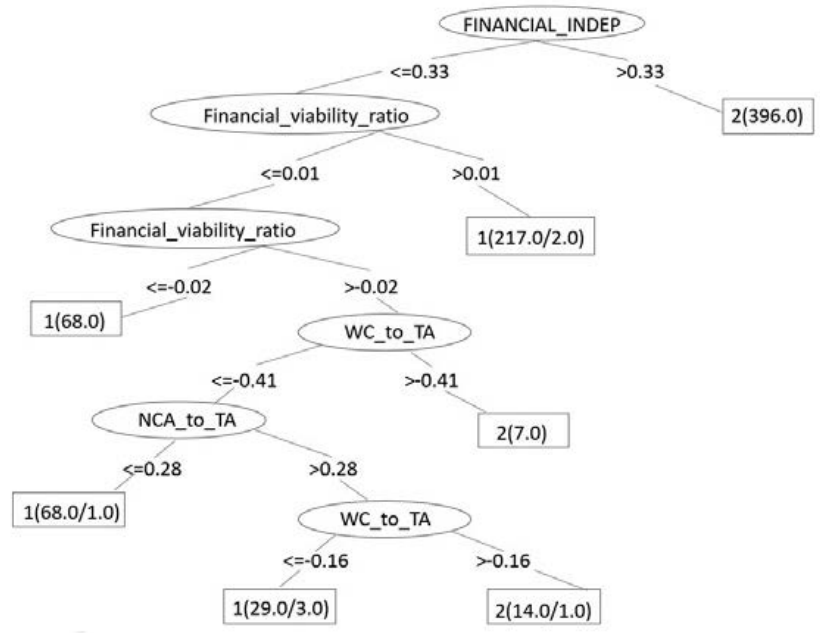

Figura 2. Resultados del árbol C4.5 para las empresas sanas

Fuente: Elaboración Propia a partir del programa WEKA

Leyenda: Financial_indep= ratio de independencia financiera; Financial_viability-ratio= ratio de viabilidad financiera; WC_to_TA= Fondo de maniobra/Total de activos; NCA_to_TA= activos no corrientes/Total de activos.

A continuación se explican cada una de las ramas del árbol de decisión, teniendo en cuenta que todas las variables y los valores frontera que aparecen han sido automáticamente generados por el árbol:

-La primera rama es el ratio de independencia financiera. Todas las empresas sanas, según el criterio de solvencia, se pueden clasificar atendiendo, en primer lugar, al ratio independencia financiera, es decir, la cuantía del patrimonio neto en función del total de los recursos disponibles (activos) de la empresa para realizar su actividad. Si ese ratio es superior a 0.33, la empresa sana es viable a corto plazo, es decir, podrá pagar sus deudas a corto plazo con su activo a corto plazo. Esta situación se cumple en 396 casos de la muestra de empresas sanas, un total del $49.5 \%$ de la muestra. Sin embargo, si la empresa tiene una cuantía de sus fondos propios inferior al 33\% de sus activos, se requiere el análisis de otra variable adicional, el ratio de viabilidad financiera. Por lo tanto,

-La segunda rama es el ratio de viabilidad financiera. Si este ratio es mayor que 0.01 , las empresas sanas no son solventes en 217 casos. Si el ratio es menor o igual que -0.02, la empresa sana tampoco es solvente a corto plazo. Esta condición se cumple en 68 empresas de la muestra. Por lo tanto, las empresas cuyo ratio de independencia financiera es inferior o igual a 0.33 y el ratio de viabilidad financiera sea inferior o igual a -0.02 o mayor que 0.01 deberían iniciar directamente el proceso de liquidación "exprés" dentro de un procedimiento concursal abreviado. En cambio, si el ratio de viabilidad financiera, es decir, si los resultados antes de impuestos, intereses y amortizaciones cubren entre el $1 \%$ y el $-2 \%$ de los gastos por intereses, entonces tenemos una tercera rama del árbol de decisión.

-La tercera rama de decisión es el ratio del fondo de maniobra. Si dicho ratio es mayor que 0.41 , entonces la empresa sana es solvente y se cumple en siete casos de la muestra. Sin 
embargo, si el fondo de maniobra no cubre al menos el $41 \%$ del total de activos, entonces tendríamos que analizar una cuarta rama del árbol.

-La cuarta rama del árbol es el porcentaje de activos no corrientes sobre el total de activos, los recursos que constituyen la estructura operativa de la empresa. Si dicho porcentaje es inferior al 28\%, la empresa no es solvente. Se hace necesario iniciar rápidamente una solución al problema de insolvencia de la empresa. Si ese porcentaje es superior al $28 \%$ de activos fijos sobre el total de los recursos, será el ratio del fondo de maniobra el que determine la solvencia de las empresas, obteniéndose una quinta y última rama del árbol.

-La quinta rama del árbol es, de nuevo, el ratio del fondo de maniobra. Si dicho ratio es inferior o igual a -0.16 , la empresa no es solvente. Si el ratio del fondo de maniobra es superior a -0.16 , la empresa sana es solvente y no tendría problemas de liquidez a corto plazo.

En cuanto al árbol de decisión de las empresas en concurso de acreedores, en la Figura 3 se observa que con solo una rama, una sola variable (al igual que en el árbol anterior tanto la variable seleccionada como el valor frontera han sido automáticamente establecidos por el software), se pueden clasificar las empresas solventes e insolventes con un elevado porcentaje de clasificación (superior al $98 \%$ en validación cruzada).

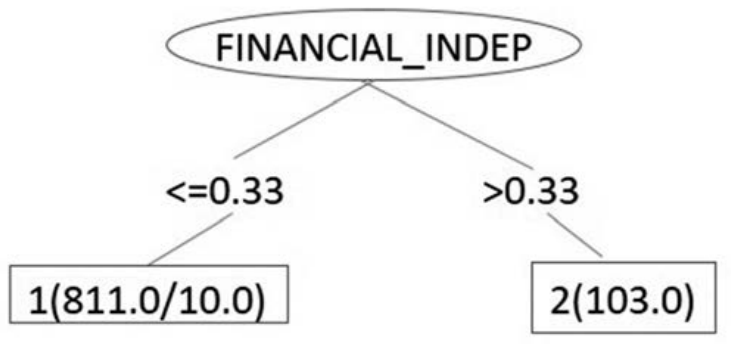

Figura 3. Resultados del árbol C4.5 para las empresas en concurso Fuente: Elaboración Propia a partir del programa WEKA

Leyenda: Financial-indep= ratio de independencia financiera.

Si el ratio de independencia financiera es inferior o igual a 0.33 , las empresas en concurso son insolventes. Esto significa que las empresas cuya cuantía del patrimonio neto sea inferior o igual al 33\% sobre el total de sus activos deberían liquidarse. Esto se cumple para 811 empresas de la muestra con 10 errores. Si la cuantía del patrimonio neto es superior al 33\% del total de activos, las empresas en concurso serían las candidatas ideales para una reorganización previa en la figura del preconcurso. Se cumple en 103 empresas de la muestra utilizada.

\section{Conclusiones e implicaciones prácticas}

El objetivo de este estudio es conocer qué características de las empresas están asociadas al éxito de su reorganización en una situación de crisis, con la finalidad de "diagnosticar" en qué 
empresas sería efectivo el preconcurso y en cuáles el preconcurso es una "situación de coma inducido". Según la muestra utilizada, cualquier tercero interesado en analizar la efectividad de una situación de preconcurso, de manera rápida y sencilla, debería calcular el valor de dos variables claves: el ratio de viabilidad financiera y el ratio del fondo de maniobra. En función de la cifra obtenida se puede discriminar si el preconcurso tendría éxito o sería una dilación innecesaria en el tiempo de un problema de muy difícil solución.

Los resultados obtenidos tienen implicaciones interesantes para los terceros interesados en la viabilidad de las empresas con problemas financieros. La resolución de este problema podría readaptarse a cualquier otra legislación concursal a nivel internacional, por ejemplo, México, donde la LMC establece una situación previa al concurso. Si una empresa se está planteando comunicar al juez su deseo de iniciar actuaciones preconcursales, los implicados en dicho proceso tendrían un primer diagnóstico sobre el posible éxito del preconcurso. Por ejemplo, si una empresa posee ratio de fondo de maniobra superior a 0.4 y su EBDITA puede cubrir el $33 \%$ de sus gastos por intereses, tiene muchas probabilidades de conseguir una reorganización viable. Esto supondría un elevado ahorro en tiempo, costes y recursos tanto públicos (judiciales, sobre todo) y privados. Además, los gestores de las empresas podrían avalar con este tipo de herramientas la reorganización de sus empresas de una manera objetiva ante acreedores y, sobre todo, ante las entidades financieras. La utilidad de los resultados del algoritmo también se puede extender a otros "stakeholders" de la empresa. En particular, los inversionistas de la empresa pueden adoptar decisiones de inversión o desinversión teniendo en cuenta las variables que condicionarían la solvencia o insolvencia de la empresa. Por ejemplo, si los administradores consideran que una ampliación de capital podría evitar que la empresa entrara en concurso de acreedores, sus inversores presentes o futuros, en función de los resultados del algoritmo, podrían avalar o rechazar dicha ampliación. También sería útil para aquellos inversionistas que buscan invertir en empresas con problemas económicos, con el fin de obtener elevadas rentabilidades en sectores en expansión o con una elevada exposición al riesgo, por ejemplo. Del mismo modo, una vez conocidas las variables claves de la reorganización empresarial de las empresas, los acreedores podrían alargar sus procesos de cobro o conceder períodos de carencia en los pagos a aquellas empresas que cumplan las características señaladas por el algoritmo y que evitarían su entrada en el proceso judicial.

Consecuentemente con lo expuesto anteriormente, también aumentaría de este modo la eficiencia del proceso judicial en general porque las empresas que fueran viables se reorganizarían y las que no, se liquidarían de manera casi automática. De este modo se evitarían las elevadas costas de los administradores judiciales, el colapso de los juzgados mercantiles y la demora en el cobro a los acreedores, entre otras. Recordemos que en la crisis económica actual muchas empresas viables económicamente han tenido que entrar en un proceso concursal por el "efecto dominó", es decir, empresas que obteniendo beneficios tenían problemas de liquidez porque otras empresas de las que eran acreedoras no les podían pagar sus créditos pendientes.

Sin embargo, este trabajo no está exento de limitaciones. La muestra es limitada, referida solamente a una comunidad autónoma de España (Madrid). Se requieren de más estudios en este sentido para avalar nuestros resultados. Como futuras líneas de investigación se plantea ampliar la muestra con empresas de otros países y en diferentes momentos de tiempo, para así poder generalizar nuestras conclusiones. 


\section{Annex: Example weka code for application J.48 (C4.5)}

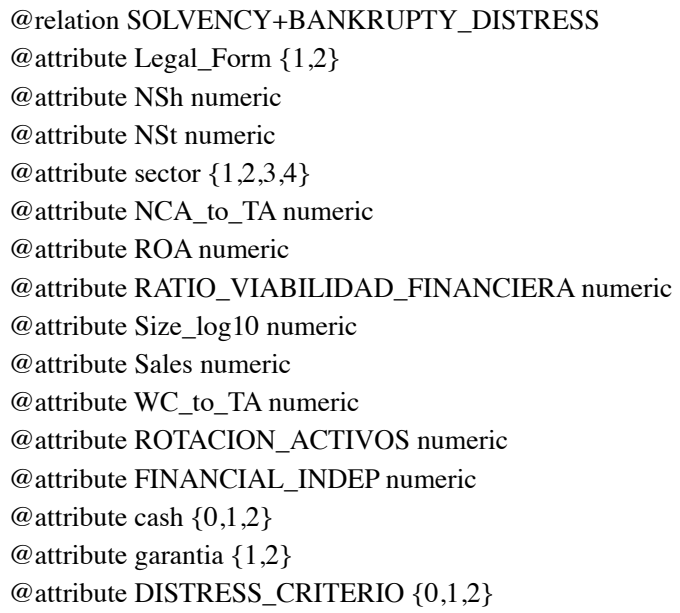

\section{Referencias}

Abellán, J. \& Mantas, C. J. (2014). Improving experimental studies about ensembles of classifiers for bankruptcy prediction and credit scoring. Expert Systems with Applications, 41 (8), 3825-3830. https://doi.org/10.1016/j. eswa.2013.12.003

Altman, E.I., Iwanicz-Drozdowska, M., Laitinen, E.K. \& Suvas, A. (2016). Financial distress prediction in an international context: a review and empirical analysis of Altman's z-score model. Journal of International Financial Management \& Accounting, 27(2), 1-41. https://doi.org/10.1111/jifm.12053

Bauweraerts, J. (2016). Predicting bankruptcy in private firms: towards a stepwise regression procedure. International Journal of Financial Research, 7(2), 147-153. https://doi.org/10.5430/ijfr.v7n2p147

Beraho, E.K. (2010). The history and evolution of bankruptcy as an international strategic management tool. International Journal of Strategic Change Management, 2 (1), 1-17. https://doi.org/10.1504/IJSCM.2010.032519

Bhirmani, A., Gulamhussen, M.A. \& Lopes, S.D. (2010). Accounting and non-accounting determinants of default: an analysis of privately-held firms. Journal of Accounting and Public Policy, 29 (6), 517-532. https://doi.or$\mathrm{g} / 10.1016 / \mathrm{j}$.jaccpubpol.2010.09.009

Bouckaert, R. R., Frank, E., Hall, M., Kirkby, R., Reutemann, P., Seewald, A. y Scuse, D. (2015). WEKA manual for version 3-7-12. New Zealand: University of Waikato.

Bryan, D. M.; Tiras, S.L. \& Wheatley, C. M. (2002). The interaction to solvency with liquidity and its association with bankruptcy emergence. Journal of Business, Finance and Accounting, 29 (7/8), 935-965. https://doi. org/10.1111/1468-5957.00456

Camacho Miñano, M. M., Segovia Vargas, M. J., \& Pascual Ezama, D. (2015). Which Characteristics Predict the Survival of Insolvent Firms? An SME Reorganization Prediction Model. Journal of Small Business Management, 53(2), 340-354. https://doi.org/10.1111/jsbm.12076

Chaudhuri, A. (2013). Bankruptcy prediction using bayesian, hazard, mixed logit and rough bayesian models: a comparative analysis. Computer and Information Science, 6 (2), 103-125. https://doi.org/10.5539/cis.v6n2p103

Chen, M.Y. (2011). Bankruptcy predictions in firms with statistical and intelligent techniques and a comparison of evolutionary computation approaches. Computers and Mathematics with Applications, 62 (12), 4514-4524. https:// doi.org/10.1016/j.camwa.2011.10.030 
Contreras, G.; Segovia-Vargas, M.J. \& Camacho-Miñano, M.M. (2015): Análisis de quiebra empresarial: modelo de ecuaciones de estimación generalizadas sobre datos panel. Tesis doctoral UCM. http://eprints.sim.ucm. es/37377/1/T37123.pdf

Díaz, Z.; Sanchis, A. \& Segovia, M.J. (2009). Analysis of Financial Instability by Means of Decision Trees and Lists, Bailly, R.O. (ed.) in Emerging Topics in Macroeconomics, NY: Editorial Nova Publishers, 303-327.

Du Jardin, P. (2015). Bankruptcy prediction using terminal failure processes. European Journal of Operational Research, 242(1), 286-303. https://doi.org/10.1016/j.ejor.2014.09.059

Fernández, A. I. (2004). La reforma concursal: ¿un diseño eficiente?. Universia Business Review, 2, 94-103.

Fernández del Pozo, L. (2010). El régimen jurídico preconcursal de los acuerdos de refinanciación (d.ad.4ㄴㄷ). Propuesta de reforma legislativa. Cuadernos de Derecho Registral. Madrid: Colegio de Registradores de la Propiedad, Fundación Registral.

Georgescu, I., \& Baciu, E. C. (2014). The Effect of Economic Crisis on Eastern European Insolvency. Procedia Economics and Finance, 15, 784-791. https://doi.org/10.1016/S2212-5671(14)00506-1

Gutiérrez, P.A., Segovia-Vargas, M.J., Salcedo-Sanz S., Hervás-Martínez, C., Sanchis A.D., Portilla-Figueras J.A. \& Fernández-Navarro, F. (2010). Hybridizing Logistic Regression with Product Unit and RBFnetworks for accurate detection and prediction of banking crises, OMEGA, 38 (5), 333-344. https://doi.org/10.1016/j.omega.2009.11.001

Hernández-Arranz, M. (2009). Fase preconcursal (art. 5.3 LC): los acreedores también podrían beneficiarse en algunos casos. Disponible en: http://www.unive.es/default.asp?menu=laboratorio\&id=83 [20 enero 2016]

Koyuncugil, A.S. \& Ozgulbas, N. (2012). Financial early warning system model and data mining application for risk detection. Expert Systems with Applications, 39 (6), 6238-6253. https://doi.org/10.1016/j.eswa.2011.12.021

Krivogorsky, V. Grudnitski, G. \& Dick, W. (2011). Bank debt and performance of Continental European firms. International Journal of Economics and Business Research, 3 (6), 593-608. https://doi.org/10.1504/IJEBR.2011.043055

Kumar, P.R. \& Ravi, V. (2007). Bankruptcy prediction in banks and firms via statistical and intelligent techniques- A review. European Journal of Operation Research, 180 (1), 1-28. https://doi.org/10.1016/j.ejor.2006.08.043

Laporta, R., López de Silanes, F., Shleifer, A. \& Vishny, R.W., (1998). Law and finance. Journal of Political Economy, 106 (6), 1113-1155. https://doi.org/10.1086/250042

Lee, S.H., Yamakawa, Y., Peng, M.W. \& Barney, J.B., (2011). How do bankruptcy laws affect entrepreneurship development around the world? Journal of Business Venturing, 26 (5), 505-520. https://doi.org/10.1016/j.jbusvent.2010.05.001

Levine R. (1998). The legal environment banks and long run economic growth. Journal of Money, Credit and Banking, 30(3), 596-613. https://doi.org/10.2307/2601259

Mongrut Montalván, S., Alberti Delgado, F.I., O’Shee D.F. \& Akamine Yamashiro, M. (2011). Determinantes de la insolvencia empresarial en el Perú. Academia, Revista Latinoamericana de Administración, 47, 126-139.

Olivencia Ruiz, M. (2015). Concurso y preconcurso. Revista de derecho concursal y paraconcursal: Anales de Doctrina, Praxis, Jurisprudencia y Legislación, 22, 11-18.

Pindado, J., Rodriguez, L., \& de la Torre, C. (2008). How do insolvency codes affect a firm's investment?. International Review of Law and Economics, 28(4), 227-238. https://doi.org/10.1016/j.irle.2008.08.001

Pulgar Ezquerra, J. (2011). Acuerdos de refinanciación y Escudos Protectores en la reforma de la LC española 22/2003. Diario LA LEY, 7731, 1-6.

Quinlan, J.R. (1993). C4.5: Programs for Machine Learning. Morgan Kaufmann: California.

Quinlan, J. R. (2014). C4. 5: programs for machine learning. Elsevier.

Rivero Romero, J. (2009) Análisis de estados financieros. Madrid: Editorial Edisofer.

Rojas Vertiz, R.M. (2002). El nuevo concurso mercantil en México. Boletín Mexicano de Derecho Comparado, No. 105, septiembre-diciembre. Disponible en: http://biblio.juridicas.unam.mx/revista/DerechoComparado/numero/105/el/el12.htm [20 enero 2016]

Rose-Green, E. \& Lovata, L. (2013). The relationship between firms' characteristics in the periods prior to bankruptcy filing and bankruptcy outcome. Accounting and Finance Research, 2 (1), 97-109. https://doi.org/10.5430/afr. v2n1p97 
Saez-Santurtun Prieto, J. (2010). Efectos de la legislación concursal en la crisis empresarial: luces y sombras. Anales de la Academia Matritense del Notariado, 50, 327-354.

Sanchis, A., Segovia, M.J.; Gil, J.A., Heras, A. \& Vilar, J.L. (2007). Rough sets and the role of the monetary policy in financial stability (macroeconomic problem) and the prediction of insolvency in insurance sector (microeconomic problem. European Journal of Operational Research, 181(3), 1554-1573. https://doi.org/10.1016/j. ejor.2006.01.045

Sathyadevan, S. \& Nair, R. R. (2015). Comparative Analysis of Decision Tree Algorithms: ID3, C4. 5 and Random Forest. In Computational Intelligence in Data Mining, 1, 549-562. Springer India.

Serrano-Cinca, C. \& Gutiérrez-Nieto, B. (2013). Partial least square discriminant analysis for bankruptcy prediction. Decision Support Systems, 54 (3), 1245-1255. https://doi.org/10.1016/j.dss.2012.11.015

Tascón, M. \& Castaño, F.J. (2012). Variables y modelos para la identificación y predicción del fracaso empresarial: revisión de la investigación empírica reciente. Revista de Contabilidad, 15(1), 7-58.https://doi.org/10.1016/S11384891(12)70037-7

Watkins, K. (2003). ¿Previeron las empresas mexicanas la crisis financiera de 1995-1996? Un análisis de empresas. El Trimestre Económico, LXX (1), Ene - Mar, 81-107.

Weijs, R. J. (2012). Harmonisation of european insolvency law and the need to tackle two common problems: common pool and anticommons. International Insolvency Review, 21(2), 67-83. https://doi.org/10.1002/iir.1197

Witten, I. H. \& Frank, E. (2005). Data mining: practical machine learning tools and techniques, 2nd edition. SanFrancisco: Morgan Kaufmann.

Wu, W.W. (2010). Beyond business failure prediction. Expert Systems with Applications, 37 (3), 2371-2376. https:// doi.org/10.1016/j.eswa.2009.07.056 